\title{
ESTIMATING SINGLE FACTOR JUMP DIFFUSION INTEREST RATE MODELS
}

\author{
Ghulam Sorwar ${ }^{1}$ \\ University of Nottingham \\ Nottingham University Business School \\ Jubilee Campus \\ Wallaton Road, Nottingham \\ NG8 1BB \\ Email: Ghulam.Sorwar@nottingham.ac.uk \\ Tele: $+44(0) 1159515487$
}

\begin{abstract}
Recent empirical studies have demonstrated that behaviour of interest rate processes can be better explained if standard diffusion processes are augmented with jumps in the interest rate process. In this paper we examine the performance of both linear and nonlinear one factor CKLS model in the presence of jumps. We conclude that empirical features of interest rates not captured by standard diffusion processes are captured by models with jumps and that the linear CKLS model provides sufficient explanation of the data.
\end{abstract}

Keywords: term structure, jumps, Bayesian, MCMC

JEL: C11, C13, C15, C32

${ }^{1}$ Corresponding author. Email Ghulam.Sorwar@nottingham.ac.uk 


\section{INTRODUCTION}

Accurate valuation of fixed income derivative securities is dependent on the correct specification of the underlying interest rate process driving it. This underlying interest rate is modelled as stochastic process comprising of two components. The first component is the drift associated with the interest rates. This drift incorporates the mean reversion that is observed in interest rates. The second component consists of a gaussian term. To incorporate heteroskedasticity observed in interest rates, the gaussian term is multiplied with the short term interest rate raised to a particular power. Different values of this power leads to different interest rate models. Setting this power to zero yields the Vasicek model (1977), setting this power to half yields the Cox-Ingersoll-Ross model (1985b), setting this power to one and half yields the model proposed by Ahn and Gao (1999). All of these one factor models are generalised by the Chan-Karolyi-LongstaffSanders model (1992,CKLS). In the CKLS model this power is unrestricted

Large number of empirical studies have demonstrated that the CKLS models or a particular variant of it, does not adequately explain the observed characteristics of interest rates, for example CKLS model ignores the possibility of non-linear drift as found by Ait-Sahalia(1996) in the case of Eurodollar rates. Furthermore, researchers have augmented the single factor CKLS model with a second or/and third stochastic factor. For example, Jegadeesh and Pennacci(1996) augment the CKLS model with stochastic mean reversion. Ball and Torus (1999) and Andersen and Lund (1997) use stochastic volatility as the second factor. However, they note, "it remains difficult to duplicate the fat-tailed or non-Gaussian innovations,." Ahn, Dittimar and Gallant (2002) test a series of 
three factor affine and quadratic models and conclude, " none of the models are able to capture the ARCH and non-Gaussian features of the observed data".

An alternative to the multi-factor approach is to augment interest rate models with jumps. This has been done by Ahn and Thompson (1988), Das (2002) and Johannes (2003).In particular Das incorporates jumps into the Vasicek model and finds strong evidence of jumps in the daily Federal Funds rate. Johannes uses a non-parametric diffusion model to study the secondary three month Treasury bills. He concludes that jumps are generally generated by the arrival of news about the macroeconomy.

In this paper we take a parametric approach in contrast to Johannes who uses a nonparametric approach to model the interest rate drift. We examine the role of jumps in a single factor CKLS framework both with linear and non-linear drift. In contrast to Johannes who assumes the jumps are log-normally distributed and the average size of jump is zero, we assume jumps are normally distributed and the average jump size is not zero. We focus on the updated data series used by Johannes. Although the period used is different from Johannes, it nonetheless includes all the major periods 1979-1981, 19911993 and 1998-1999 that Johannes studies in depth. In order to better understand the role played by jumps we estimate the unobserved jump times and jump sizes.

In summary this paper provides strong evidence that CKLS model incorporated with jumps provides a better description of data than a CKLS model without jumps. For all the three separate periods considered we find that diagnostic tests based on residual analysis indicates that CKLS without jumps is mis-specified.. We also find that the difference in performance between a CKLS model with linear and non-linear drift is negligible once jumps have been incorporated. Thus we can conclude linear drift plus 
jump provides sufficient description of the data. Finally data augmentation leads to the same conclusion.

The remaining part of this paper comprises of the following sections. Section 2 introduces the models and the estimation procedure. Section 3 summarises the empirical results. Section 4 concludes this paper.

\section{JUMP DIFFUSION MODEL AND PARAMETER ESTIMATION PROCEDURE}

The general one factor interest rate model comprises of a linear drift with a constant elasticity of variance. As a stochastic process, it is stated as:

$d r_{t}=\kappa\left(\theta-r_{t}\right) d t+\sigma r_{t}^{\gamma} d W_{t}$

Parameters in the above model have intuitive interpretation. $\theta$ is the central tendency parameter for the interest rate $r$ which mean reversion at rate $\kappa$. The variance coefficient of the diffusion is given by $\sigma^{2}$, whilst the variance of elasticity is given by $2 \gamma$. Setting $\gamma=$ 0 yields the Vasicek; setting $\gamma=1 / 2$ yields the CIR model, an unrestricted value of yields the CKLS model. Simple version of this model has been found to be highly unsatisfactory, as a result Ait-Sahalia (1996) has proposed a more flexible model that can incorporate non-linear drift and a more general form of the diffusion coefficient. He proposes the following diffusion process:

$d r_{t}=\left[\kappa\left(\theta-r_{t}\right)+\alpha_{2} r_{t}^{2}+\frac{\alpha_{3}}{r_{t}}\right] d t+\sqrt{\beta_{0}+\beta_{1} r_{t}+\beta_{2} r_{t}^{\beta_{3}}} d W_{t}$

Conley, Hansen, Luttmer, and Scheinkman (1997,CHLS) maintain the same drift as AitSahalia (1996) but simplified the diffusion term to CEV process used by CKLS:

$$
d r_{t}=\left[\kappa\left(\theta-r_{t}\right)+\alpha_{2} r_{t}^{2}+\frac{\alpha_{3}}{r_{t}}\right] d t+\sigma r_{t}^{\gamma} d W_{t}
$$


To ensure stationarity $\alpha_{2}<0$ and $\alpha_{3}>0$. For the remaining part of this study we add a random jump term to the short rate equation:

$d r_{t}=\left[\kappa\left(\theta-r_{t}\right)+\alpha_{2} r_{t}^{2}+\frac{\alpha_{3}}{r_{t}}\right] d t+\sigma r_{t}^{\gamma} d W_{t}+\xi d N_{t}$

The term $\xi d N_{t}$ represents the jump component. $N_{\mathrm{t}}$ is a Poisson process with constant intensity $\lambda$ and $\xi$ is the jump size in interest rates. We assume that $\xi$ is normally distributed $\xi \sim N\left(\mu_{J}, \sigma_{J}^{2}\right)$. Naturally imposing $\lambda=0$ ensures no jumps are present..

Finally for convenience we define $\alpha_{0}$ as $\kappa \theta$ and $\alpha_{1}$ as $-\kappa$ to yield

$$
d r_{t}=\left(\alpha_{0}+\alpha_{1} r_{t}+\alpha_{2} r_{t}^{2}+\frac{\alpha_{3}}{r_{t}}\right) d t+\sigma r_{t}^{\gamma} d W_{t}+\xi d N_{t}
$$

For estimation purposes we use the first order Euler approximation of equation as given by

$$
r_{n+1}=r_{n}+\left(\alpha_{0}+\alpha_{1} r_{n}+\alpha_{2} r_{n}^{2}+\frac{\alpha_{3}}{r_{n}}\right) \Delta t+\sigma r_{n}^{\gamma} \varepsilon \sqrt{\Delta t}+\xi_{n+1} J_{n+1}
$$

In the above discretisation, the Gaussian term is approximated by $\varepsilon \sqrt{\Delta t}$ where $\varepsilon$ is normally distributed with mean of zero and variance of one. Further we assume that at most a single jump can occur over each time interval with $P\left[J_{n}=1\right]=\lambda \in(0,1)$.

Equation (6) with data augmentation if required is used as the basis for Gibbs or Metropolis-Hasting (MH) sampling (see Eraker (2001, 2003), Jones (1999,2002) for recent applications of Markov Chain Monte Carlo (MCMC) to financial time series) . The steps involved are:

1. Initialise $r, \alpha_{0}, \alpha_{1}, \alpha_{2}, \alpha_{3}, \sigma^{2}, \lambda, \gamma, J, \xi$

2. Choose number of extra points in between actual data if data augmentation used. 
3. Sample $r_{t}$ given $r_{n} \mid r_{\backslash n}, \alpha_{0}, \alpha_{1}, \alpha_{2}, \alpha_{3}, \sigma^{2}, \lambda, \gamma, J, \xi$

4. Using normal prior sample $\left(\alpha_{0}, \alpha_{1}, \alpha_{2}, \alpha_{3}\right) \mid r, \sigma^{2}, \lambda, \gamma, J, \xi$

5. Using inverse gamma prior sample $\sigma^{2} \mid r, \alpha_{0}, \alpha_{1}, \alpha_{2}, \alpha_{3}, \lambda, \gamma, J, \xi$

6. Using normal prior sample $\gamma \mid r, \alpha_{0}, \alpha_{1}, \alpha_{2}, \alpha_{3}, \sigma^{2}, \lambda, J, \xi$

7. Using beta prior sample $\lambda \mid r, \alpha_{0}, \alpha_{1}, \alpha_{2}, \alpha_{3}, \sigma^{2}, \gamma, J, \xi$

8. Using Bernoulli prior sample $J \mid r, \alpha_{0}, \alpha_{1}, \alpha_{2}, \alpha_{3}, \sigma^{2}, \gamma, \lambda, \xi$

9. Using normal prior sample $\xi \mid \alpha_{0}, \alpha_{1}, \alpha_{2}, \alpha_{3}, \sigma^{2}, \gamma, \lambda, J$

All the steps except step 3 and step 6 which uses MH sampling uses Gibbs sampling. Cycling through steps 1 to 9 represents one complete sweep of the sampler. For data augmentation we follow the procedure in Jones (1999). We need to perform thousands of such sweeps to estimate the model parameters.

The ability of the different models to fit the observed data can be assessed by examining the normalised residuals that are generated in the estimation process. The residuals are based on the Euler approximation, for example for the one factor interest rates, the residuals are:

$$
\frac{r_{n+1}-r_{n}-\left(\alpha_{0}+\alpha_{1} r_{n}+\alpha_{2} r_{n}^{2}+\frac{\alpha_{3}}{r_{n}}\right) \Delta t-\xi_{n+1} J_{n+1}}{\sigma_{n}^{\gamma} \sqrt{\Delta t}}=\varepsilon_{n+1} \approx N(0,1)
$$

In the Euler approximation the normalised residuals are assumed to be independent standard normal random variables. According to Zellner (1975) $\varepsilon$ may be interpreted as a parameter vector and hence its posterior distributions of various functions may be computed. To assess the viability of the models moments and autocorrelations should be 
calculated. If there is any misspecification in the model then independence or normality will be violated.

Posterior distributions of these functions are obtained in the same manner to posteriors of the model parameters. Thus at each iteration we first calculate the model parameters and then based on the model parameters we calculate the series of residual $\varepsilon_{n+1}$.

\section{EMPIRICAL RESULTS}

The three month secondary market quote T- bill rates are used in this study. As noted by a number of researchers the three month T- bills have the advantages of high liquidity, small bid ask spreads and are free of idiosyncratic effects that may lead to potential sources of non-normality. Johannes (2003) notes that omitting weekends and holidays has no impact on the conclusions which can be reached using daily data. Thus in this study we use daily data from January 1979 to December 2002. The total number of observations is 5994 .

Table 2 - Table 4 contain parameters for three separate sub time period based on daily observations. Finally Table 5 contains parameters for one sub time period with data augmentation.

Table 2 contains the parameters for the period 1/2/1979 to 12/31/1981. During this period the jump intensity is around $18 \%$ for the linear models and around $19 \%$ for the non-linear models indicating a period of large frequent jumps. The average jump size is positive and $\gamma$ is always greater than 1. Without the jumps $\gamma$ is just over one, however, with the jumps $\gamma$ is always greater 1.5 for linear and non-linear models. The $\alpha_{3}$ parameter is small with and without jumps indicating that the linear model is sufficient. Without the 
jumps the skewness and the kurtosis is around 0.1 and 5.6 respectively for both models. Introduction of jumps brings these statistics to acceptable levels around 0 and 3.

Table 3 covers the period $1 / 2 / 1991$ to $12 / 31 / 1992$. During this period the jumps are smaller. The average jump intensity for this period drops to around $7 \%$. The average jump size is negative. Unlike the previous period $\gamma$ is close to 0 . However the addition of jumps raises $\gamma>0.5$ for both linear and non-linear models. In the case of non-linear models with jumps $\alpha_{2}>0$ indicating potential mis-specification. Futher $\alpha_{3}$ drops significantly once jumps are introduced, indicating that jumps eliminate any non-linearity that may be present in the interest rate. The skewness, kurtosis and correlation are greater than 3,14 and 0.1 respectively indicating very strong mis-specification without the jumps.

Table 4 covers the period $1 / 2 / 1998$ to $12 / 29 / 2000$. During this period $\gamma$ is approximately zero both with and without jumps, in contrast to the previous two periods. The average jump size is negative. Unlike the previous two periods there is a large difference in jump intensity between the linear models and the non-linear models. For linear models jump intensity is around $10 \%$ whereas for the non-linear models jump intensity is around $4 \%$. Introducing jumps has no significant impact on $\alpha_{3}$. The skewness and kurtosis parameter again indicate strong mis-specificatin without the jumps.

In Table 5, we again estimate the parameters for the period 1/2/1991 to $12 / 31 / 1992$, in this case with data augmentation. Furthermore we only focus on the linear models as the conclusions drawn using non-linear models would be the same. The first feature to note is the impact on the specification statistics for the models without the jumps. Even with 
$\mathrm{t}=1 / 2$, that is with 2 simulated extra data points inbetween actual observations the specification statistics are at the boundary of acceptability. With $\Delta t=1 / 8$, i.e. with 8 simulated data points between actual observations, the specification statistics are wholly acceptable. With jumps incorporated the specification statistics are always acceptable. Overall data augmentation does not cause the parameters $\alpha_{0}, \alpha_{1}, \sigma^{2}, \gamma, \delta^{2}$ to be significantly different from those obtained without any data augmentation. The average jump size is negative although increasing the number of simulated data points causes it to decrease in absolute terms. The jump intensity is naturally lower with data augmentation as we have effectively more periods with data augmentation.

Figure 2 - Figure 5 contain plots for the three different sub-periods. Each figure comprises of 5 separate plots. The first plot exhibits the daily change in the actual interest rate. The second plot shows the daily probabilities of a jump. The third plot shows the daily average jump sizes. Both the second and the third models are based on linear models. The final two plots are based on the non-linear models and show daily jump probabilities and average jump sizes respectively.

From Figure 2 we see that for the period 1979-1982 one factor linear and non-linear CKLS with jumps is able to predict both the times and the magnitudes of the jumps. However, a point to note is that the magnitude of the predicted jump size is smaller than the actual observed jump size. For example, the largest observed jump was 134 basis points. The corresponding model jump size based on the linear model is 102 basis points and based on the non-linear model it is 99 basis points. Thus clearly for the period both the linear and non-linear models underestimate the magnitude of the jumps. 
Figure 3 covers the period 1991-1992. As can be seen from the first plot the daily changes in this sub-period is much smaller than the previous sub-period. For example, during this period the largest daily change in interests rate was a fall of 31 basis points. For this period the estimated jump sizes are more accurate than the previous period. For example the corresponding jump size based on the linear model was -29 basis points and based on the non-linear model it was also -29 basis points. During this sub-period Johannes (2003) identifies the following event dats; 01/09/1991, outbreak of the Gulf War; 02/01/1991, unemployment announcements and comments by the Federal Reserve; 08/19/1991, the Kremlin Coup and the Collapse of the Soviet Union; 08/21/1991, the emergence of Boris Yeltsin as the leader of Russia; 12/20/1991, the Federal Reserve lowered the discount rate. All these events are reproduced by both the linear and the nonlinear CKLS model with probabilities of $60 \%$ or more.

Figure 4 covers the period 1998-2000. The jumps during this period are similar in magnitude to the previous period. The largest observed jump is -49 basis points. The corresponding jumps based on the linear and non-linear CKLS models are -46.5 and -47 basis points respectively.

Thus in summary when the jump sizes are of the order of 50 basis points, the estimated jump sizes can be calculated with a very high accuracy, however as during the first subperiod 1979-1982 where the jump sizes are larger than 130 basis points, there may be a small discrepancy between the actual and estimated jump sizes.

Figure 5 covers the period 1991-1992 with data augmentation. Figure 8 can be directly compared with Figure 3. In terms of appearance, the two figures are similar. However, closer examination indicates that once data augmentation is used both the probabilities 
and the jump sizes decrease in absolute value and there are many more smaller jumps around the actual date of jump.

\section{CONCLUSIONS}

In this paper we have examined the CKLS (1992) model augmented with normally distributed jumps. Our results indicate that this model accurately captures the tail behaviour of interest. Furthermore, we firstly find the non-linear drift offers no overall advantage over the linear drift and secondly the linear drift is correctly specified in contrast to the non-linear drift for certain periods. We also estimate the model parameter using data augmentation and find that overall our conclusions remain the same. However, with data augmentation, the average jump sizes predicted by the models are smaller.

In this paper we have demonstrated that jumps are an important component in models explaining the empirical properties of interest rates. The next step is to examine multi-factor CKLS models with jumps in the short rate and the long rate. 


\section{REFERENCES}

Ahn, C. M. and H. E. Thomson (1988), 'Jump-Diffusion Process and Term Structure of Interest Rates,' Journal of Finance, 43, 155-174.

Ahn, D. and B. Gao (1999), 'A Parametric Nonlinear Model of Term Structure Dynamics,' Review of Financial Studies, 12, 721-762.

Ahn, D., R. Dittimar and A. R. Gallant (2002), 'Quadratic Term Structure Models: Theory and Evidence,' Review of Financial Studies, 15, 243-288.

Aït-Sahalia, Y. (1996) 'Testing Continuous-Time Models of the Spot Interest Rate,' Review of Financial Studies ,9, 385-426.

Andersen, T. and J. Lund (1997), 'Estimating Continuous-Time Stochastic Volatility Models of the Short-Term Interest Rate,' Journal of Econometrics, 77, 343-377. Ball, C., and W. N. Torus (1983), 'A Simplified Jump Process for Common Stock Returns,' Journal of Financial and Quantitative Analysis, 18, 53-65.

Ball, C., and W. N. Torus (1999), 'The Stochastic Volatility of Short-Term Interest Rates: Some International Evidence,' Journal of Finance, 54, 2339-2359. Chan, K.C., G.A. Karolyi, F.A. Longstaff and A.B. Sanders (1992) 'An Empirical Comparison of the Short-Term Interest Rate,' Journal of Finance, 47, 1209-1227. Conley, T.G., L.P. Hansen, E.G.J Luttmer and J.A. Scheikman (1997), 'Short-Term Interest Rates as Subordinated Diffusions,' Review of Financial Studies, 10, 525-578.

Cox, J. C., J. E. Ingersoll and S. A. Ross (1985a), 'An Intertemporal General Equilibrium Model of Asset Prices,' Econometrica, 53, 363-384.

Cox, J. C., J. E. Ingersoll and S. A. Ross (1985b), 'A Theory of the Term Structure of Interest Rate,' Econometrica, 53, 385-407.

Das, S.R. (2002), 'The Surprise Element: Jumps in Interest Rates,' Journal of Econometrics, 106, 27-65.

Eraker, B. (2001), 'MCMC Analysis of Diffusion Models with Application to Finance,' Journal of Business and Economic Statistics, 19, 177-191.

Jegadeesh, N. and G. G. Pennacci (1996), 'The Behaviour of Interest Rates Implied by the Term Structure of Eurodollar Futures,' Journal of Money Credit and Banking, 28, 426-446.

Johannes, M.S. (2003), 'The Statistical and Economic Role of Jumps in Interest Rates,' Journal of Finance, forthcoming.

Jones, C.S. (1999), 'Bayesian Estimation of Continuous-Time Finance Models,' working paper, University of Rochester.

Jones, C.S. (2002), 'The Dynamics of Stochastic Volatility: Evidence from Underlying and Options Market,'working paper, University of Rochester.

Vasicek, O. (1977), 'An Equilibrium Characterization of the Term Structure Modeling,' Journal of Financial Economics, 5, 177-188.

Zellner, A. (1975), 'Bayesian Analysis of Regression Error,' Journal of the American Statistical Association, 70, 138-144. 
Table 1: Treasury bill rate Summary Statistics from January 1979 to December 2002

\begin{tabular}{llllllll}
\hline \hline & Mean & Min & Max & SD & Skew & Kurt & AC \\
\hline$r_{t}(\%)$ & 6.56 & 1.14 & 17.14 & 3.06 & 0.94 & 0.83 & 0.99 \\
$r_{t+\Delta}(\%)$ & $-1.37 \times 10^{-5}$ & -0.127 & 0.0134 & $11.76 \times 10^{-4}$ & 0.38 & 22.99 & 0.13 \\
\hline
\end{tabular}


Table 2: Treasury bill parameter estimates, 1/2/1979 to 12/31/1981 based on daily observations. For each parameter $I$ report the mean of the posterior distribution and the standard deviation of the posterior in parentheses. The mean, standard deviation, skewness, kurtosis and first autocorrelation is given in the lower part of the table. For each statistic the mean and standard deviation is calculated, with the standard deviation in parentheses.

\begin{tabular}{|c|c|c|c|c|}
\hline & LIN & LINJ & NLIN & NLINJ \\
\hline $1000 \times \alpha_{0}$ & $0.5083(0.3783)$ & $0.7138(0.3267)$ & $-32.208(15.0428)$ & $-38.9943(23.8296)$ \\
\hline$\alpha_{1}$ & $-0.0041(0.0035)$ & $-0.0068(0.00309)$ & $0.3168(0.1449)$ & $0.3668(0.2156)$ \\
\hline$\alpha_{2}$ & & & $-1.0048(0.4497)$ & $-1.1317(0.6342)$ \\
\hline$\alpha_{3}$ & & & $0.0011(0.0005)$ & $0.0014(0.0009)$ \\
\hline $10 \times \sigma^{2}$ & $0.0089(0.0043)$ & $0.02918(0.0341)$ & $0.0085(0.0039)$ & $0.0511(0.0760)$ \\
\hline$\gamma$ & $1.1365(0.1072)$ & $1.5108(0.2093)$ & $1.1270(0.1012)$ & $1.6124(0.2346)$ \\
\hline $1000 \times \xi$ & & $0.6667(0.4899)$ & & $0.4083(0.5061)$ \\
\hline $1000 \times \delta^{2}$ & & $0.0168(0.0039)$ & & $0.0160(0.0038)$ \\
\hline$\lambda$ & & $0.1793(0.0379)$ & & $0.1873(0.0382)$ \\
\hline \multicolumn{5}{|c|}{ Specification Analysis } \\
\hline $\operatorname{Mean}\left(\varepsilon_{t}^{r}\right)$ & $0.0000(0.0364)$ & $-0.0002(0.0366)$ & $-0.0001(0.0370)$ & $-0.0002(0.0367)$ \\
\hline $\operatorname{StDev}\left(\varepsilon_{t}^{r}\right)$ & $0.9995(0.0261)$ & $0.9995(0.0261)$ & $0.9994(0.0255)$ & $0.9996(0.0258)$ \\
\hline $\operatorname{Skew}\left(\varepsilon_{\mathrm{t}}^{\mathrm{r}}\right)$ & $0.1300(0.0088)$ & $0.0085(0.0123)$ & $0.1081(0.0267)$ & $0.0084(0.0125)$ \\
\hline $\operatorname{Kurt}\left(\varepsilon_{t}^{r}\right)$ & $5.6342(0.0293)$ & $3.2762(0.1932)$ & $5.5837(0.0503)$ & $3.2470(0.1950)$ \\
\hline$\rho\left(\varepsilon_{t}^{\mathrm{r}}\right)$ & $0.1568(0.0046)$ & $0.0895(0.0296)$ & $0.1612(0.0058)$ & $0.0900(0.0296)$ \\
\hline
\end{tabular}


Table 3: Treasury bill parameter estimates, $1 / 2 / 1991$ to $12 / 31 / 1992$ based on daily observations. For each parameter I report the mean of the posterior distribution and the standard deviation of the posterior in parentheses. The mean, standard deviation, skewness, kurtosis and first autocorrelation is given in the lower part of the table. For each statistic the mean and standard deviation is calculated, with the standard deviation in parentheses.

\begin{tabular}{lllll}
\hline \hline & LIN & LINJ & NLIN & NLINJ \\
\hline $1000 \times \alpha_{0}$ & $0.0963(0.0619)$ & $0.1146(0.0560)$ & $-8.7579(4.4268)$ & $-0.5546(4.3791)$ \\
$\alpha_{1}$ & $-0.0037(0.0014)$ & $-0.0035(0.0013)$ & $0.1976(0.1054)$ & $0.0019(0.1045)$ \\
$\alpha_{2}$ & & $-1.4729(0.8162)$ & $0.03490(0.8099)$ \\
$1000 \times \alpha_{3}$ & & $0.1249(0.0605)$ & $0.0149(0.0598)$ \\
$10000 \times \sigma^{2}$ & $0.0087(0.0083)$ & $0.0726(0.1011)$ & $0.0083(0.0073)$ & $0.0584(0.0717)$ \\
$\gamma$ & $0.1932(0.1056)$ & $0.5983(0.1809)$ & $0.1892(0.1048)$ & $0.55706(0.1917)$ \\
$1000 \times \xi$ & & $-0.3807(0.2544)$ & & $-0.4555(0.2902)$ \\
$10000 \times \delta^{2}$ & & $0.0129(0.0048)$ & & $0.0135(0.0058)$ \\
$\lambda$ & & $0.0789(0.0252)$ & $0.0731(0.0249)$ \\
\hline \hline & & Specification Analysis & \\
\hline $\operatorname{Mean}\left(\varepsilon_{\mathrm{t}}^{\mathrm{r}}\right)$ & $-0.0029(0.0442)$ & $-0.0009(0.0447)$ & $0.0002(0.0447)$ & $0.0000(0.0449)$ \\
$\operatorname{StDev}\left(\varepsilon_{\mathrm{t}}^{\mathrm{r}}\right)$ & $0.9992(0.0317)$ & $0.9996(0.0317)$ & $0.9997(0.0316)$ & $0.9990(0.0317)$ \\
$\operatorname{Skew}\left(\varepsilon_{\mathrm{t}}^{\mathrm{r}}\right)$ & $3.2349(0.2388)$ & $0.0205(0.0243)$ & $3.1348(0.2627)$ & $0.0200(0.0232)$ \\
$\operatorname{Kurt}\left(\varepsilon_{\mathrm{t}}^{\mathrm{r}}\right)$ & $14.5631(0.5459)$ & $3.4820(0.2310)$ & $14.0121(0.6102)$ & $3.4663(0.2231)$ \\
$\rho\left(\varepsilon_{\mathrm{t}}^{\mathrm{r}}\right)$ & $0.1270(0.0031)$ & $0.0957(0.0247)$ & $0.1299(0.0050)$ & $0.0935(0.0257)$ \\
\hline
\end{tabular}


Table 4: Treasury bill parameter estimates, $1 / 2 / 1998$ to $12 / 29 / 2000$ based on daily observations. For each parameter I report the mean of the posterior distribution and the standard deviation of the posterior in parentheses. The mean, standard deviation, skewness, kurtosis and first autocorrelation is given in the lower part of the table. For each statistic the mean and standard deviation is calculated, with the standard deviation in parentheses.

\begin{tabular}{lllll}
\hline \hline & LIN & LINJ & NLIN & NLINJ \\
\hline $1000 \times \alpha_{0}$ & $0.2145(0.1336)$ & $0.1395(0.0901)$ & $-85.2941(19.7088)$ & $-98.2444(16.3505)$ \\
$\alpha_{1}$ & $-0.0040(0.0026)$ & $-0.0025(0.0018)$ & $1.6837(0.3960)$ & $1.9104(0.3274)$ \\
$\alpha_{2}$ & & $-10.9923(2.6298)$ & $-12.2916(2.1712)$ \\
$\alpha_{3}$ & & $0.0014(0.0003)$ & $0.0017(0.0003)$ \\
$10000 \times \sigma^{2}$ & $0.0035(0.0008)$ & $0.0016(0.0013)$ & $0.0035(0.0008)$ & $0.0024(0.0017)$ \\
$\gamma$ & $0.0293(0.0293)$ & $0.0675(0.0676)$ & $0.0326(0.0312)$ & $0.0681(0.0677)$ \\
$1000 \times \xi$ & & $-0.0274(0.1871)$ & & $-0.4280(0.4574)$ \\
$1000 \times \delta^{2}$ & & $0.0019(0.0006)$ & & $0.0396(0.0223)$ \\
$\lambda$ & & $0.1011(0.0282)$ & $0.0436(0.0178)$ \\
\hline \hline \multicolumn{5}{c}{ Specification Analysis } \\
\hline $\operatorname{Mean}\left(\varepsilon_{\mathrm{t}}^{\mathrm{r}}\right)$ & $-0.0013(0.0365)$ & $-0.0017(0.0364)$ & $0.0002(0.0367)$ & $-0.0001(0.0363)$ \\
$\operatorname{StDev}\left(\varepsilon_{\mathrm{t}}^{\mathrm{r}}\right)$ & $0.9999(0.0260)$ & $0.9995(0.0256)$ & $0.9997(0.0256)$ & $0.9993(0.0255)$ \\
$\operatorname{Skew}\left(\varepsilon_{\mathrm{t}}^{\mathrm{r}}\right)$ & $0.7023(0.0541)$ & $0.0099(0.0143)$ & $1.1589(0.1370)$ & $0.0133(0.0155)$ \\
$\operatorname{Kurt}\left(\varepsilon_{\mathrm{t}}^{\mathrm{r}}\right)$ & $26.7870(0.1310)$ & $3.5532(0.2311)$ & $28.4467(0.6359)$ & $3.8104(0.1790)$ \\
$\rho\left(\varepsilon_{\mathrm{t}}^{\mathrm{r}}\right)$ & $0.0462(0.0038)$ & $0.1153(0.0292)$ & $0.0627(0.0077)$ & $0.1348(0.0232)$ \\
\hline
\end{tabular}


Table 5: Treasury bill parameter estimates, $1 / 2 / 1991$ to $12 / 31 / 1992$ based on daily observations with data augmentation. Time steps of $\Delta t=1 / 2$ and $\Delta t=1 / 8$ are used. For each parameter $I$ report the mean of the posterior distribution and the standard deviation of the posterior in parentheses. The mean, standard deviation, skewness, kurtosis and first autocorrelation is given in the lower part of the table. For each statistic the mean and standard deviation is calculated, with the standard deviation in parentheses.

\begin{tabular}{lllll}
\hline \hline & LIN & LINJ & LIN & LINJ \\
\hline \multicolumn{2}{c}{$\Delta \mathrm{t}=1 / 2$} & \multicolumn{3}{c}{$\Delta \mathrm{t}=1 / 8$} \\
\hline $1000 \times \alpha_{0}$ & $0.0952(0.0618)$ & $0.1132(0.0556)$ & $0.0950(0.0624)$ & $0.1161(0.0563)$ \\
$\alpha_{1}$ & $-0.0037(0.0014)$ & $-0.0035(0.0013)$ & $-0.0037(0.0014)$ & $-0.0035(0.0013)$ \\
$10000 \times \sigma^{2}$ & $0.0662(0.0510)$ & $1.2910(1.6475)$ & $0.0525(0.0277)$ & $0.5620(0.5558)$ \\
$\gamma$ & $0.1570(0.0972)$ & $0.6943(0.1930)$ & $0.1343(0.0762)$ & $0.6104(0.1408)$ \\
$1000 \times \xi$ & & $-0.2597(0.1840)$ & & $-0.2188(0.1561)$ \\
$10000 \times \delta^{2}$ & & $0.096(0.0390)$ & $0.0791(0.0300)$ \\
$\lambda$ & $0.0405(0.0150)$ & $0.0169(0.0068)$ \\
\hline \hline $\operatorname{Mean}\left(\varepsilon_{t}^{\mathrm{r}}\right)$ & $-0.0019(0.0257)$ & $-0.0000(0.0259)$ & $-0.0011(0.0149)$ & $-0.0002(0.0147)$ \\
$\operatorname{StDev}\left(\varepsilon_{t}^{\mathrm{r}}\right)$ & $0.9999(0.0184)$ & $0.9997(0.0181)$ & $0.9999(0.0105)$ & $1.0000(0.0105)$ \\
$\operatorname{Skew}\left(\varepsilon_{\mathrm{t}}^{\mathrm{r}}\right)$ & $0.1177(0.0447)$ & $0.0042(0.0060)$ & $0.0056(0.0052)$ & $0.0014(0.0019)$ \\
$\operatorname{Kurt}\left(\varepsilon_{\mathrm{t}}^{\mathrm{r}}\right)$ & $4.2207(0.2550)$ & $3.0369(0.1292)$ & $3.1356(0.0867)$ & $3.0038(0.0732)$ \\
$\rho\left(\varepsilon_{\mathrm{t}}^{\mathrm{r}}\right)$ & $0.0145(0.0255)$ & $0.0089(0.0257)$ & $0.0016(0.0150)$ & $0.0007(0.0148)$ \\
\hline
\end{tabular}


Figure 1: The time series of the daily-level and changes (in basis points) in the three-month Treasury bill rate from January 1979 - December 2002

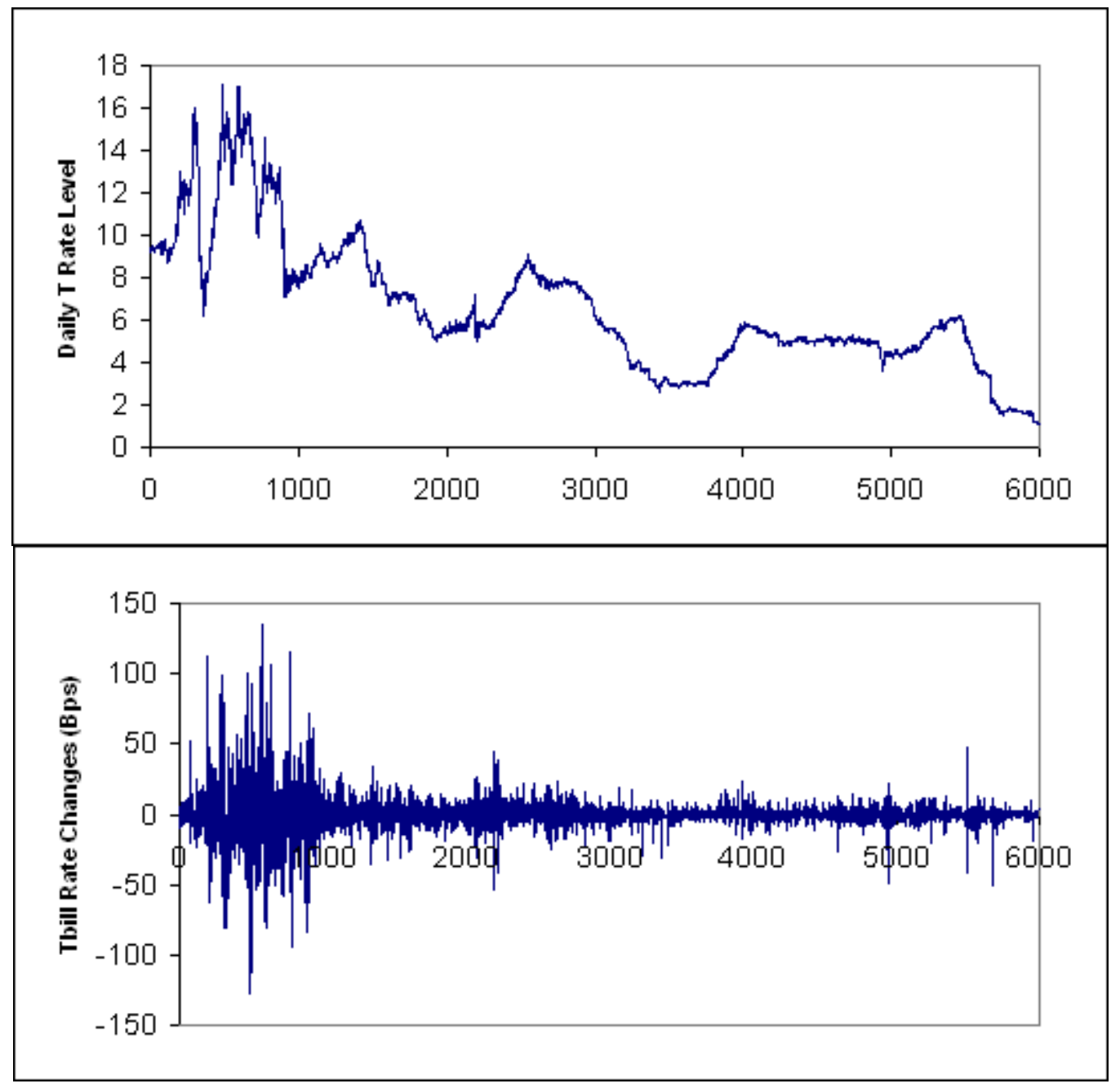


Figure 2: Time series interest rate changes, estimated jump probabilities and jump sizes for linear and non-linear drift from 1979-1982
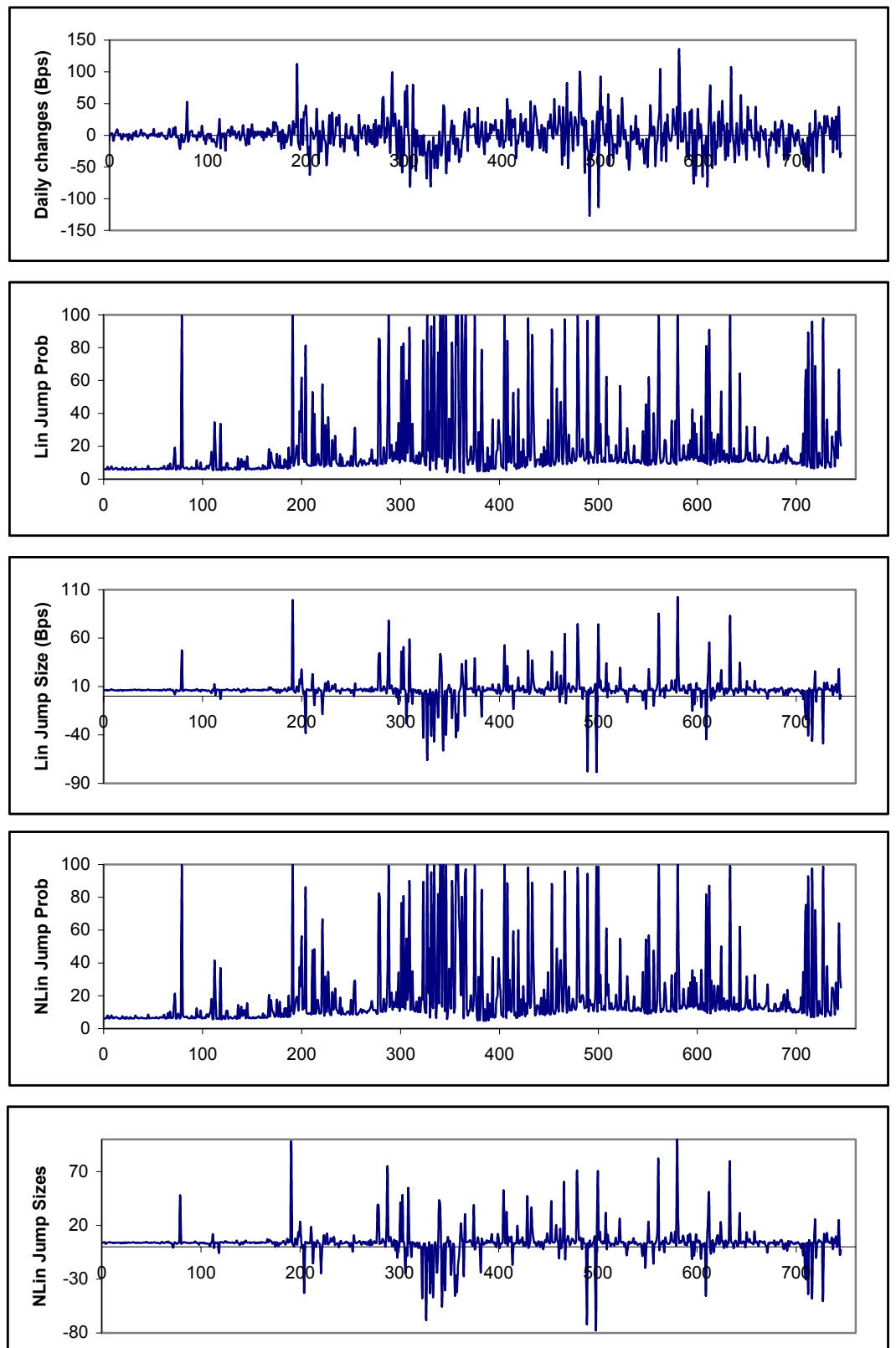
Figure 3: Time series interest rate changes, estimated jump probabilities and jump sizes for linear and non-linear drift from 1991-1992
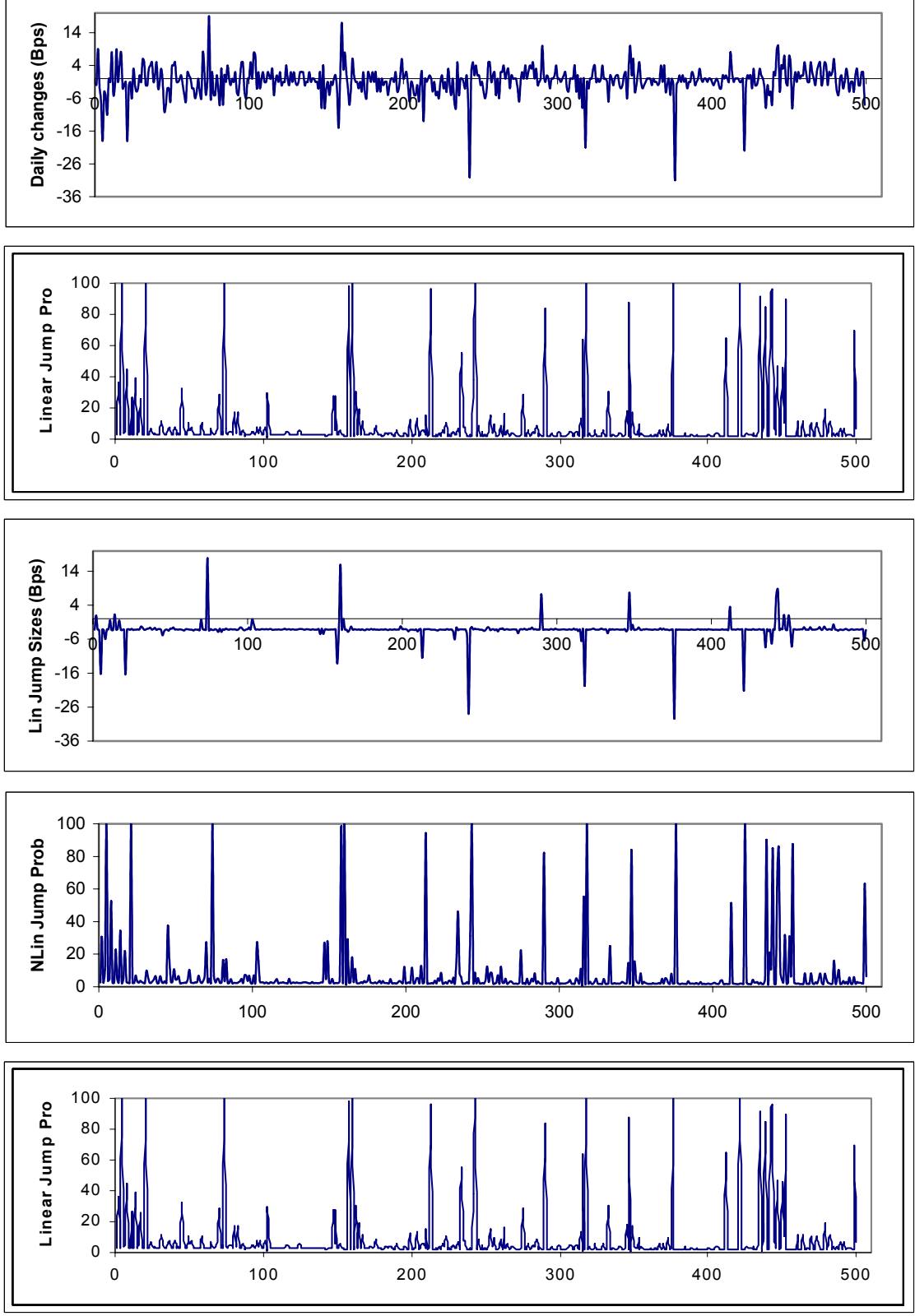
Figure 4: Time series interest rate changes, estimated jump probabilities and jump sizes for linear and non-linear drift from 1998-2000.
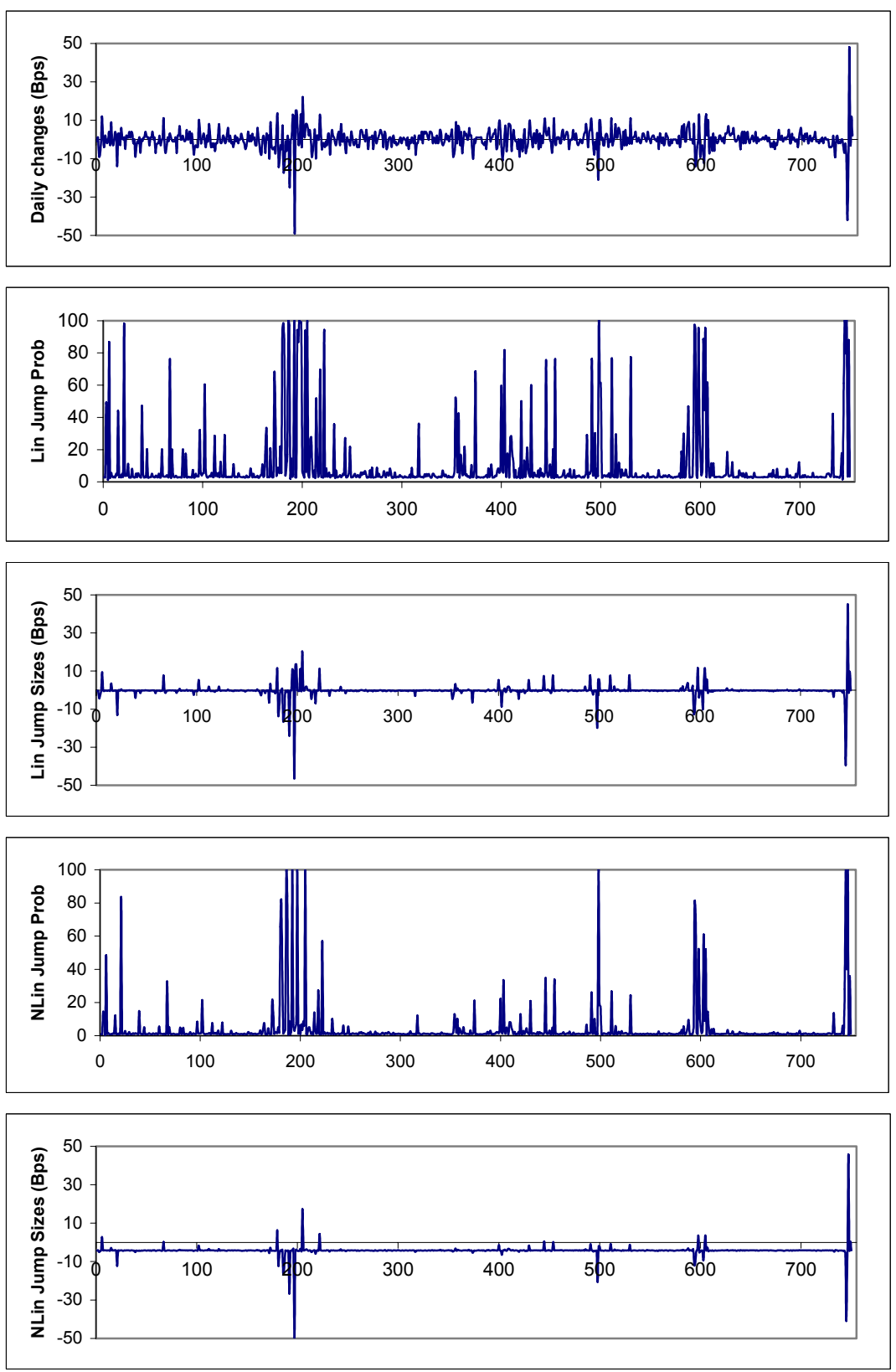
Figure 5: Time series interest rate changes, estimated jump probabilities and jump sizes for linear and non-linear drift from 1991-1992 for $h=1 / 2$ and $h=1 / 8$.
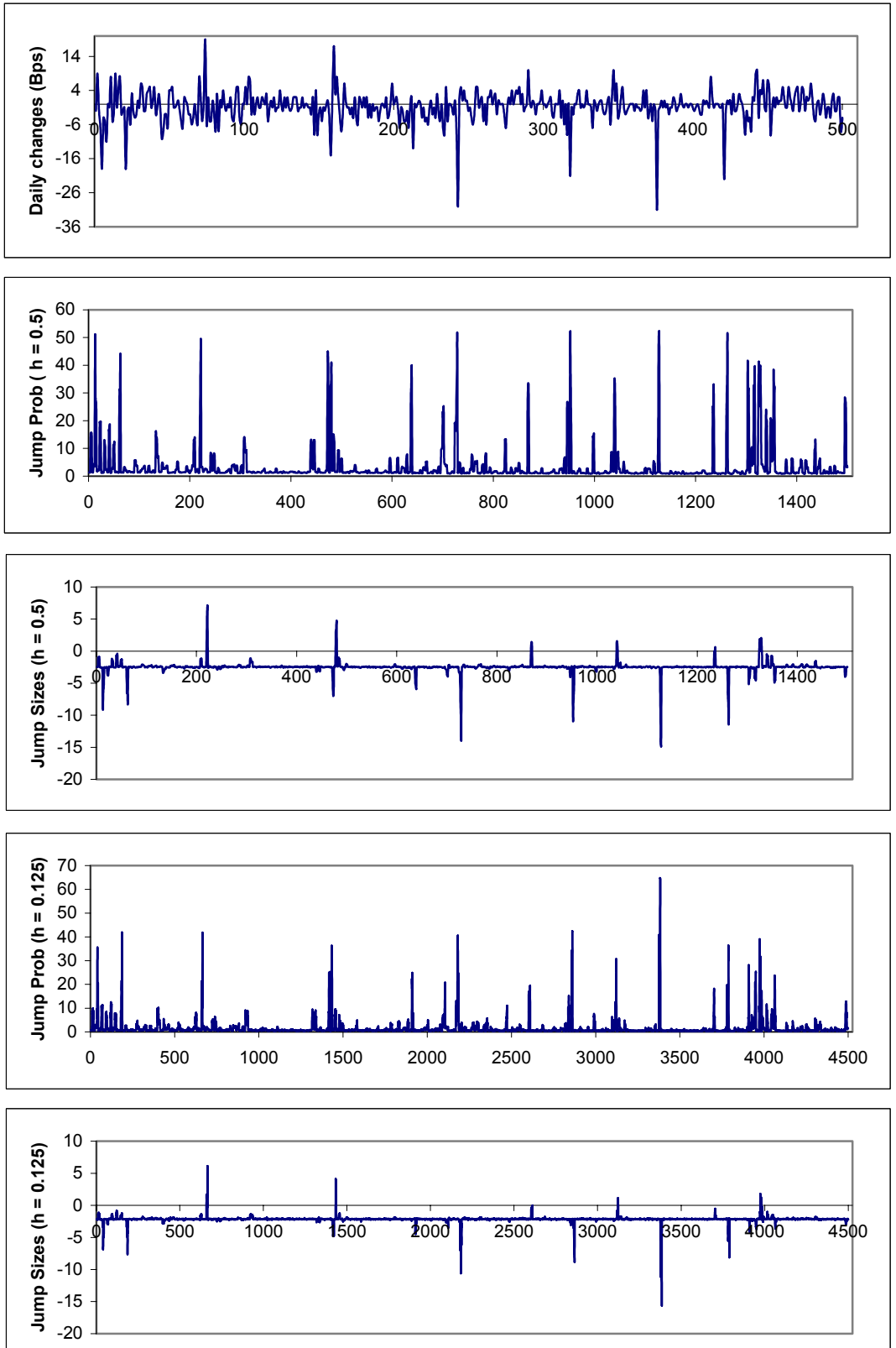